\begin{tabular}{|c|l|}
\hline Title & A nisotropic Superconductivity in Highly Disordered Systems \\
\hline Author(s) & Toyama, Takahiro; Ohkawa, Fusay oshi J. \\
\hline Citation & $\begin{array}{l}\text { Journal of the Physical Society of Japan, 78(9), 094712 } \\
\text { https://doi.org/10.1143/PSJ.78.094712 }\end{array}$ \\
\hline Issue Date & 2009-09-15 \\
\hline Doc URL & http://hdl.handle.net/2115/39309 \\
\hline Rights & ○ 2009 The Physical Society of Japan \\
\hline Type & article (author version) \\
\hline File Information & 63777physb-style.pdf \\
\hline
\end{tabular}

Instructions for use 


\title{
Anisotropic Superconductivity in Highly Disordered Systems*
}

\author{
Takahiro Toyama ${ }^{\dagger}$ AND Fusayoshi J. OHKAWA \\ Department of Physics, Graduate School of Science, Hokkaido University, Sapporo Japan 060-0801
}

(Received April 24, 2009; accepted June 24, 2009; published September 10, 2009)

\begin{abstract}
The gap equation for anisotropic superconductivity is solved in the presence of elastic scatterings by nonmagnetic and magnetic impurities, which are treated by the self-consistent Born approximation, and inelastic scatterings, which are phenomenologically treated. When elastic scatterings are strong, even at $T=0 \mathrm{~K}$, coherence peaks almost disappear and the gap is a pseudogap, i.e., the density of states $\rho(\varepsilon)$ is nonzero at the chemical potential, which means that the $T$-linear specific heat coefficient is nonzero in the superconducting state. At $T=0 \mathrm{~K}$ in such a case, the low-energy part of $\rho(\varepsilon)$ or the gap spectrum has a concave-cap V shape, which is in contrast to a convex-cup $\mathrm{V}$ shape in the absence of scattering. When elastic or inelastic scatterings are strong, the ratio $\epsilon_{G}(0) / k_{\mathrm{B}} T_{c}$ is much larger than its mean-field value of about 4 , where $\epsilon_{G}(0)$ is the gap at $T=0 \mathrm{~K}$ and $T_{c}$ is the superconducting critical temperature. The large $\epsilon_{G}(0) / k_{\mathrm{B}} T_{c} \simeq 8$ and the linear decrease in $T_{c}$ in residual resistivity, both of which are observed in cuprate superconductors and the latter of which is inconsistent with the Abrikosov and Gor'kov theory, can be explained by the temperature-dependent pair breaking estimated from the $T$-linear coefficient of resistivity, which is about $1 \mu \Omega \mathrm{cm} / \mathrm{K}$.
\end{abstract}

Keywords: anisotropic superconductivity, disorder, AG theory, cuprate oxide

\section{Introduction}

High-temperature superconductivity in cuprate oxide is an interesting and important issue in solid-state physics. ${ }^{1-6}$ Many issues on it remain contentious despite the two decades that has already passed since its discovery. A parent cuprate is a highly anisotropic quasi-two-dimensional compound composed of $\mathrm{CuO}_{2}$ planes. When not doped, it is an antiferromagnet below the Néel temperature $T_{N}$, which is within 200-500 $\mathrm{K}$ depending on the cuprate, and is a Mott insulator above $T_{N}$. The Néel state is stabilized by the superexchange interaction between nearest neighbor $\mathrm{Cu}$ ions, which is as strong as $J=-(0.10-0.15) \mathrm{eV} .{ }^{7}$ When holes are doped, the Néel state disappears and a $d_{x^{2}-y^{2}-}$ wave superconducting state appears. ${ }^{2}$ Both $T_{N}$ and $T_{c}$ are very low or negligible for $\delta \simeq 0.05$, where $T_{c}$ is the superconducting critical temperature and $\delta$ is the concentration of doped holes per unit cell. The transition between the two phases is apparently continuous as a function of $\delta$, although magnetism and superconductivity cannot be characterized by the symmetry breaking or lowering from a phase to the other phase. Disorders certainly play a crucial role in the apparently continuous transition.

Since $T_{c}$ is highest at $\delta=\delta_{\text {opt }}$, where $\delta_{\text {opt }}=0.15-0.18$, $\delta=\delta_{\text {opt }}$ denotes optimaldoping; $\delta<\delta_{\text {opt }}$ and $\delta>\delta_{\text {opt }}$ denote underdoping and overdoping, respectively. The observed resistivity $R(T)$ at $T>T_{c}$ is approximately described by ${ }^{8,9}$

$$
R(T)=R(0)+r_{1} T
$$

The resistivity extrapolated to $T=0 \mathrm{~K}$ or the residual resistivity $R(0)$ is due to a disorder. It is large for underdoped cuprates. When $\delta \simeq 0.05$, for example, it is as large

\footnotetext{
*Published in J. Phys. Soc. Jpn. 78 (2009) 094712 (1-8). DOI: $10.1143 /$ JPSJ.78.094712

(C)The Physical Society of Japan

${ }^{\dagger}$ E-mail address: sasuke@mail.sci.hokudai.ac.jp

${ }^{\ddagger}$ E-mail address: fohkawa@mail.sci.hokudai.ac.jp
}

as $R(0) \simeq 10^{3} \mu \Omega \mathrm{cm}$. Disorders certainly suppress $d_{x^{2}-y^{2}}$ wave superconductivity in underdoped cuprates with $\delta \simeq$ 0.05 . The residual resistivity $R(0)$ is small for optimally doped cuprate oxides. Disorder is not crucial in optimally doped cuprates. The $T$-linear coefficient $r_{1}$ is large for $0.05 \lesssim \delta \lesssim \delta_{\text {opt }}$ such as

$$
r_{1} \simeq 1 \mu \Omega \mathrm{cm} / \mathrm{K}
$$

Since inelastic scatterings by superconducting fluctuations, which develop near the center of the Brillouin zone, are almost forward scatterings, their contribution to the resistivity must be small. The $T$-linear coefficient $r_{1}$ can be explained in terms of inelastic scatterings by antiferromagnetic spin fluctuations in two or highly anisotropic quasitwo dimensions. ${ }^{10}$

According to the Abrikosov and Gor'kov (AG) theory, ${ }^{11}$ the $T_{c}$ of an isotropic $s$-wave or Bardeen-Cooper-Schrieffer (BCS) superconductivity ${ }^{12}$ is not reduced by nonmagnetic impurities but is reduced by magnetic impurities. When scatterings by magnetic impurities are sufficiently strong, BCS superconductivity disappears. In the case of anisotropic $s$-wave superconductivity, the reduction in $T_{c}$ by magnetic impurities obeys the AG theory while that by nonmagnetic impurities is rather moderate and does not decrease $T_{c}$ to $0 \mathrm{~K}$ in general. ${ }^{13-17}$ In the case of purely anisotropic superconductivities such as $p$-wave and $d$-wave superconductivity, the reduction in $T_{c}$ by nonmagnetic and magnetic impurities simply obeys the AG theory, ${ }^{18}$ so that anisotropic superconductivity disappears when the scatterings by nonmagnetic or magnetic impurities are sufficiently strong.

Impurity scattering affects other properties of superconductors such as the density of states. ${ }^{19,20}$ It is interesting to determine how impurity scattering affects the density of states of $d_{x^{2}-y^{2}}$-wave superconductors. When a strong pair breaking at $T=0 \mathrm{~K}$, which is indicated by a large residual resistivity $R(0)$, is considered, it is easy to explain the disappearance of $d_{x^{2}-y^{2}}$-wave superconductivity for $\delta \lesssim 0.05$. It is interesting to examine how anisotropic superconductivity or an anisotropic superconducting gap disappears as 
the strength of the pair breaking is increased.

According to the mean-field theory, ${ }^{21,22}$ which considers no pair breaking,

$$
\epsilon_{G}(0) / k_{\mathrm{B}} T_{c} \simeq 4
$$

for a $d_{x^{2}-y^{2}}$ wave, where $\epsilon_{G}(0)$ is the superconducting gap at $T=0 \mathrm{~K}$. On the other hand, the observed ratio is as large as

$$
\epsilon_{G}(0) / k_{\mathrm{B}} T_{c} \simeq 8,
$$

for optimally doped superconductors and is larger than 8 for underdoped ones. Pair breaking due to inelastic scatterings by superconducting and antiferromagnetic fluctuations reduces $T_{c}$. Since inelastic scatterings disappear at $T=0 \mathrm{~K}$, the amount of reduction in $\epsilon_{G}(0)$ induced by them is small. When inelastic scatterings are strong, therefore, the ratio can be much larger than 4 . It is interesting to examine how large $\epsilon_{G}(0) / k_{\mathrm{B}} T_{c}$ can be when both elastic and inelastic scatterings are strong.

The purpose of this study is to determine effects of elastic and inelastic scatterings on physical properties in an anisotropic superconducting state, such as the disappearance of an anisotropic superconducting gap, the reduction in $T_{c}$, and the increase in $\epsilon_{G}(0) / k_{\mathrm{B}} T_{c}$. This paper is organized as follows. The formulation is given in $\S 2$. Results are given in $\S 3$. The application of what to the cuprate superconductor is given in $\S 4$. The conclusion is given in $\S$ 5 .

\section{Formulation}

In this paper, strong electron correlations are not discussed; it is simply assumed on the basis of previous papers $^{23,24}$ that the normal state above $T_{c}$ is a conventional Fermi liquid, which may exhibit apparently anomalous Fermi-liquid behaviors such as the $T$-linear resistivity at relatively low temperatures as well as at sufficiently high temperatures. ${ }^{10}$ Then, we consider a simple effective Hamiltonian on a quasi-two-dimensional or threedimensional lattice:

$$
\mathcal{H}=\mathcal{H}_{1}+\mathcal{H}_{2}+\mathcal{H}_{3}+\mathcal{H}_{4}
$$

The first term describes noninteracting electrons or it effectively describes quasi-particles, which are renormalized by electron correlations:

$$
\mathcal{H}_{1}=\sum_{\boldsymbol{k} \sigma} E(\boldsymbol{k}) a_{\boldsymbol{k} \sigma}^{\dagger} a_{\boldsymbol{k} \sigma} .
$$

The density of states is defined by

$$
\rho_{0}(\varepsilon)=\frac{1}{N} \sum_{\boldsymbol{k}} \delta[\varepsilon+\mu-E(\boldsymbol{k})]=\rho_{0},
$$

where $\mu$ is the chemical potential. For simplicity, it is assumed that $\rho_{0}(\varepsilon)$ is constant. The second term describes an attractive interaction between quasi-particles:

$$
\mathcal{H}_{2}=-\frac{1}{2 N} \sum_{\boldsymbol{k} \boldsymbol{p} \boldsymbol{q}} \sum_{\sigma \sigma^{\prime}} g(\boldsymbol{q}) a_{\boldsymbol{k}+\boldsymbol{q} \sigma}^{\dagger} a_{\boldsymbol{p}-\boldsymbol{q} \sigma^{\prime}}^{\dagger} a_{\boldsymbol{p} \sigma^{\prime}} a_{\boldsymbol{k} \sigma}
$$

where $N$ is the number of unit cells and $g(\boldsymbol{q})$ is an effective attractive interaction. It is assumed for simplicity that $g(\boldsymbol{q})$ decouples in such a way that

$$
g(\boldsymbol{k}-\boldsymbol{p})=\sum_{l=1}^{+\infty} g_{l}^{\mathrm{sc}} \eta_{l}(\boldsymbol{k}) \eta_{l}(\boldsymbol{p})
$$

where

$$
\eta_{l}(\boldsymbol{k})=\cos \left[l \tan ^{-1}\left(k_{x} / k_{y}\right)\right],
$$

is the form factor of the $l$ th partial wave. Note that

$$
\frac{1}{N} \sum_{\boldsymbol{k}} \eta_{l}(\boldsymbol{k})=0
$$

for any $l \geq 1$. In this study, it is assumed that the Fermi surface is isotropic; the assumption made here is relevant provided that the Fermi surface is isotropic. The third and fourth terms respectively describe scatterings by nonmagnetic and magnetic impurities:

$$
\mathcal{H}_{3}=\sum_{i \in \mathcal{R}_{n}} \sum_{\sigma} V_{i} a_{i \sigma}^{\dagger} a_{i \sigma}-\bar{V} \sum_{i \sigma} a_{i \sigma}^{\dagger} a_{i \sigma},
$$

and

$$
\mathcal{H}_{4}=\sum_{i \in \mathcal{R}_{s}} \sum_{\sigma \sigma^{\prime}} J_{i}\left(\boldsymbol{S}_{i} \cdot \boldsymbol{\sigma}^{\sigma \sigma^{\prime}}\right) a_{i \sigma}^{\dagger} a_{i \sigma^{\prime}}
$$

where

$$
a_{i \sigma}=\frac{1}{\sqrt{N}} \sum_{\boldsymbol{k}} e^{i \boldsymbol{k} \cdot \boldsymbol{R}_{i}} a_{\boldsymbol{k} \sigma}
$$

$V_{i}, \bar{V}$, and $J_{i}$ are real, $\boldsymbol{S}_{i}$ is a localized spin at the $i$ th site, and $\boldsymbol{\sigma}=\left(\sigma_{x}, \sigma_{y}, \sigma_{z}\right)$ is the Pauli matrix. In eqs. (2.8) and (2.9), the site summations over $i \in \mathcal{R}_{n}$ and $i \in \mathcal{R}_{s}$ run over nonmagnetic and magnetic impurity sites, respectively. We consider an ensemble for sets of impurities. One of the most crucial assumptions in this study is that there is no correlation among impurities at different sites, the strength of each single impurity is so weak that $\left|V_{i}\right| \rho_{0} \ll 1$ and $\left|J_{i}\right| \rho_{0} \ll 1$, and the impurity concentrations of nonmagnetic and magnetic impurities per unit cell, which are denoted by $n_{n}$ and $n_{s}$, respectively, are so dense that $\left(n_{n}+n_{s}\right)(\ell / a)^{2} \gg 1$ and $\left(n_{n}+n_{s}\right)(\xi / a)^{2} \gg 1$ in quasi-two dimensions and $\left(n_{n}+n_{s}\right)(\ell / a)^{3} \gg 1$ and $\left(n_{n}+n_{s}\right)(\xi / a)^{3} \gg 1$ in three dimensions, where $\ell$ is the mean free path of quasi-particles, $\xi$ is the superconducting coherent length, and $a$ is the lattice constant. In order to compensate for the energy shift due to impurities, the constant $\bar{V}$ is defined by ${ }^{25}$

$$
\bar{V}=\left\langle\left\langle V_{i}\right\rangle\right\rangle
$$

where $\langle\langle\cdots\rangle$ represents the average over the ensemble. Then, it follows that ${ }^{25}$

$$
\left.\left\langle\left(V_{i}-\bar{V}\right)\left(V_{j}-\bar{V}\right)\right\rangle\right\rangle=n_{n} \overline{\Delta V^{2}} \delta_{i j},
$$

where $\sqrt{\overline{\Delta V^{2}}}$ is the effective strength of nonmagnetic impurities. It is also assumed that there is no correlation among localized spins $\boldsymbol{S}_{i}$ at different sites so that $\left\langle\left\langle J_{i} \boldsymbol{S}_{i}\right\rangle_{T}=0\right.$ and $^{25}$

$$
\left\langle\left\langle J_{i} J_{j}\left(\boldsymbol{S}_{i} \cdot \boldsymbol{S}_{j}\right)\right\rangle\right\rangle_{T}=n_{s} \overline{J^{2}} S(S+1) \delta_{i j},
$$


where $\sqrt{J^{2}}$ is the effective strength of magnetic impurities and $S$ is the magnitude of their spins. Here, $\left\langle\langle\cdots\rangle_{T}\right.$ represents the average over the thermal average as well as the ensemble average.

Following the AG theory ${ }^{11}{ }^{1}$ it is straightforward to derive the gap equation

$$
\frac{1}{g_{l}^{\mathrm{sc}}}=-k_{\mathrm{B}} T \sum_{\left|\varepsilon_{n}\right|<\theta} \frac{1}{N} \sum_{\boldsymbol{k}} \eta_{l}^{2}(\boldsymbol{k}) \frac{1}{D\left(\mathrm{i} \varepsilon_{n}, \boldsymbol{k}\right)}
$$

where $\theta$ is the cutoff energy and $D\left(\mathrm{i} \varepsilon_{n}, \boldsymbol{k}\right)$ is defined by

$$
\begin{aligned}
D\left(\mathrm{i} \varepsilon_{n}, \boldsymbol{k}\right)= & {\left[\mathrm{i} \varepsilon_{n}+\mu-E(\boldsymbol{k})-\Sigma\left(\mathrm{i} \varepsilon_{n}\right)\right] } \\
& \times\left[\mathrm{i} \varepsilon_{n}-\mu+E(\boldsymbol{k})+\Sigma\left(-\mathrm{i} \varepsilon_{n}\right)\right] \\
& -\frac{1}{4} \epsilon_{G}^{2}(T) \eta_{l}^{2}(\boldsymbol{k}),
\end{aligned}
$$

where $\epsilon_{G}(T)$ is the gap parameter and $\Sigma\left(\mathrm{i} \varepsilon_{n}\right)$ is the selfenergy due to scatterings from impurities. Equation (2.7) is used in deriving the gap equation (2.14). In the selfconsistent Born approximation, self-energy is given by

$$
\Sigma\left(\mathrm{i} \varepsilon_{n}\right)=g_{\mathrm{imp}}^{2} \frac{1}{N} \sum_{\boldsymbol{k}} G\left(\mathrm{i} \varepsilon_{n}, \boldsymbol{k}\right),
$$

where

$$
g_{\mathrm{imp}}^{2}=n_{n} \overline{\Delta V^{2}}+n_{s} S(S+1) \overline{J^{2}},
$$

and

$$
G\left(\mathrm{i} \varepsilon_{n}, \boldsymbol{k}\right)=\frac{\mathrm{i} \varepsilon_{n}-\mu+E(\boldsymbol{k})+\Sigma\left(-\mathrm{i} \varepsilon_{n}\right)}{D\left(\mathrm{i} \varepsilon_{n}, \boldsymbol{k}\right)} .
$$

In cuprate superconductors, quasi-particles are also scattered by superconducting and antiferromagnetic fluctuations. The effects of inelastic scatterings are phenomenologically considered in this study. Instead of eq. (2.16),

$$
\Sigma\left(\mathrm{i} \varepsilon_{n}\right)=g_{\mathrm{imp}}^{2} \frac{1}{N} \sum_{\boldsymbol{k}} G\left(\mathrm{i} \varepsilon_{n}, \boldsymbol{k}\right)+\Delta \Sigma\left(\mathrm{i} \varepsilon_{n}\right),
$$

where $\Delta \Sigma\left(\mathrm{i} \varepsilon_{n}\right)$ is a phenomenological term, is considered. On the basis of the observation ${ }^{8,9}$ that resistivity is approximately proportional to $T$ above $T_{c}$ in cuprate superconductors and the theoretical study ${ }^{10}$ showing that antiferromagnetic spin fluctuations are responsible for the $T$-linear resistivity, it is assumed that

$$
\Delta \Sigma\left(\mathrm{i} \varepsilon_{n}\right)=-\mathrm{i} \alpha k_{\mathrm{B}} T \frac{\varepsilon_{n}}{\left|\varepsilon_{n}\right|} .
$$

This is assumed not only above $T_{c}$ but also below $T_{c}$, for simplicity. ${ }^{26}$

Assuming that the Fermi surface is isotropic, the gap equation (2.14) becomes simple so that

$$
\frac{1}{\lambda_{\mathrm{sc}}}=\int_{-\infty}^{\infty} d E \int_{0}^{2 \pi} \frac{d \phi}{2 \pi} \int_{-\theta}^{\theta} d \varepsilon f(\varepsilon) \frac{1}{\pi} \operatorname{Im} \frac{\cos ^{2}(\phi)}{D(\varepsilon+\mathrm{i} 0, E, \phi)},
$$

where $f(\varepsilon)=1 /\left[\exp \left(\varepsilon / k_{\mathrm{B}} T\right)+1\right], \phi=l \tan ^{-1}\left(k_{x} / k_{y}\right)$,

$$
\lambda_{\mathrm{sc}}=\frac{1}{2} \rho_{0} g_{l}^{\mathrm{sc}}
$$

is a dimensionless coupling constant, and

$$
\begin{aligned}
D(\varepsilon+\mathrm{i} 0, E, \phi)= & {[\varepsilon+\mu-E-\Sigma(\varepsilon+\mathrm{i} 0)] } \\
& \times[\varepsilon-\mu+E+\Sigma(-\varepsilon-\mathrm{i} 0)] \\
& -\frac{1}{4} \epsilon_{G}^{2}(T) \cos ^{2} \phi .
\end{aligned}
$$

Note that, provided that $l \geq 1$, the gap equation does not depend on $l$ because the Fermi surface is isotropic. When the gap equation is solved, the density of states is given by

$$
\rho(\varepsilon)=-\frac{1}{\pi N} \sum_{\boldsymbol{k}} \operatorname{Im}[G(\varepsilon+\mathrm{i} 0, \boldsymbol{k})] .
$$

When $\epsilon_{G}(T)=0$ or $T \geq T_{c}$, it is easy to solve eq. (2.19). It follows that

$$
\Sigma(\varepsilon+\mathrm{i} 0)=-\mathrm{i}\left(\gamma_{0}+\alpha k_{\mathrm{B}} T\right),
$$

with

$$
\gamma_{0}=\pi \rho_{0} g_{\mathrm{imp}}^{2} .
$$

There are four parameters in the theoretical framework of this paper: $\gamma_{0}$ is the strength of impurity scatterings, $\alpha$ is the strength of inelastic scatterings, $\lambda_{\mathrm{sc}}$ is the strength of the attractive interaction, and $\theta$ is the cutoff energy. When $\theta$ is considered as the unit of energy, the number of parameters is reduced to three: $\gamma_{0}, \alpha$, and $\lambda_{\mathrm{sc}}$.

\section{Results}

According to eq. (2.21), $T_{c}$ for $\alpha=0$ and $\gamma_{0}=0$ or $T_{c}$ in the presence of no pair breaking, which is denoted by $T_{c 0}$, is given by

$$
\frac{k_{\mathrm{B}} T_{c 0}}{\theta}=\frac{2}{\pi} e^{\gamma_{E}} e^{-1 / \lambda_{\mathrm{sc}}}=1.1338 \cdots \times e^{-1 / \lambda_{\mathrm{sc}}},
$$

where $\gamma_{E}=0.57721 \cdots$ is the Euler constant. This is simply the BCS theory. Since $T_{c 0}$ can also be used as a parameter instead of $\lambda_{\mathrm{sc}}$, the three parameters are $\gamma_{0}, \alpha$, and either $\lambda_{\mathrm{sc}}$ or $T_{c 0}$.

When $\alpha=0$ and $\gamma_{0} \neq 0, T_{c}$ is given by

$$
\ln \frac{T_{c 0}}{T_{c}}=\psi\left(\frac{\gamma_{0}}{2 \pi k_{\mathrm{B}} T_{c}}+\frac{1}{2}\right)-\psi\left(\frac{1}{2}\right),
$$

where $\psi(z)$ is the di-gamma function. This is simply the AG theory. According to eq. (3.2), $T_{c}=0 \mathrm{~K}$ for $\gamma_{0} \geq \gamma_{\mathrm{AG}}$, where $\gamma_{\mathrm{AG}}$ is defined by

$$
\frac{\gamma_{\mathrm{AG}}}{k_{\mathrm{B}} T_{c 0}}=\frac{\pi}{2} e^{-\gamma_{E}}=0.88193 \cdots .
$$

Figure 1 shows $T_{c} / T_{c 0}$ as a function of $\gamma_{0} / k_{\mathrm{B}} T_{c 0}$ for various $\alpha$ values including $\alpha=0$. When $\alpha$ is nonzero, $T_{c}$ is suppressed from $T_{c 0}$, which is for $\alpha=0$ and is given by eq. (3.2). Since $\alpha k_{\mathrm{B}} T$ vanishes as $T \rightarrow 0 \mathrm{~K}$, the critical $\gamma_{0}$ at which $T_{c}$ becomes zero is independent of $\alpha$ and is given by eq. (3.3) for any $\alpha$. Note that $T_{c}$ decreases almost linearly with $\gamma_{0}$ when $\alpha \gtrsim 1$. 


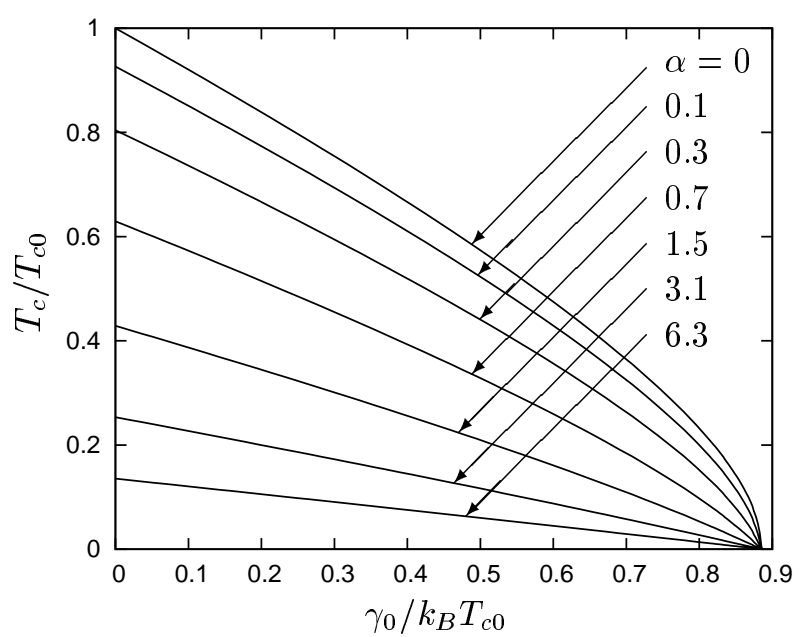

FIG. 1: $T_{c} / T_{c 0}$ vs $\gamma_{0} / k_{\mathrm{B}} T_{c 0}$ for $\alpha=0,0.1,0.3,0.7,1.5,3.1$, and 6.3 .

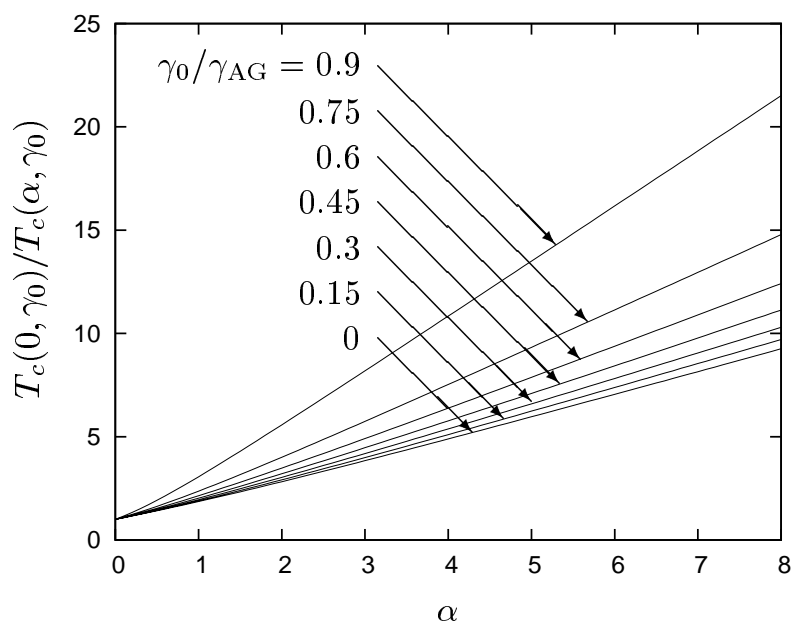

FIG. 2: $T_{c}\left(0, \gamma_{0}\right) / T_{c}\left(\alpha, \gamma_{0}\right)$ vs $\alpha$ for $\gamma_{0} / \gamma_{\mathrm{AG}}=0,0.15,0.3,0.45$, $0.6,0.75$, and 0.9 .

Figure 2 shows $T_{c}\left(\alpha=0, \gamma_{0}\right) / T_{c}\left(\alpha, \gamma_{0}\right)$ as a function of $\alpha$ for various $\gamma_{0}$ values. There is a quantitative difference between the case of $\gamma_{0} \ll \gamma_{\text {AG }}$ and the case of $\gamma_{0} \simeq \gamma_{\mathrm{AG}}$. When $\gamma_{0} / \gamma_{\mathrm{AG}} \lesssim 0.3$,

$$
\frac{T_{c}\left(\alpha=0, \gamma_{0}\right)}{T_{c}\left(\alpha, \gamma_{0}\right)} \simeq 1+\alpha .
$$

When $\gamma_{0} / \gamma_{\mathrm{AG}}<1$ and $\gamma_{0} / \gamma_{\mathrm{AG}} \simeq 1$,

$$
\frac{T_{c}\left(\alpha=0, \gamma_{0}\right)}{T_{c}\left(\alpha, \gamma_{0}\right)} \simeq 1+2 \alpha \text {. }
$$

Figure 3 shows $\epsilon_{G}(0) / k_{\mathrm{B}} T_{c}$ as a function of $\gamma_{0} / \gamma_{\mathrm{AG}}$ for various $\lambda_{\mathrm{sc}}$ values and $\alpha=0$. It should be mentioned that $\epsilon_{G}(0) / k_{\mathrm{B}} T_{c}$ depends on not only $\gamma_{0}$ but also $\lambda_{\mathrm{sc}}$. The dependence of $\epsilon_{G}(0) / k_{\mathrm{B}} T_{c}$ on $\lambda_{\mathrm{sc}}$ is quite weak for a small $\lambda_{\mathrm{sc}}$ such as $\lambda_{\mathrm{sc}} \lesssim 0.3 ; \epsilon_{G}(0) / k_{\mathrm{B}} T_{c} \simeq 4.3$ in the limit of $\lambda_{\mathrm{sc}} \rightarrow 0$. The weak dependence is a strong-coupling effect, which is partly due to the anisotropy of the gap. When $\gamma_{0} / \gamma_{\mathrm{AG}} \lesssim 0.5$

$$
\frac{\epsilon_{G}(0)}{k_{\mathrm{B}} T_{c}} \simeq 4+2 \frac{\gamma_{0}}{\gamma_{\mathrm{AG}}}
$$

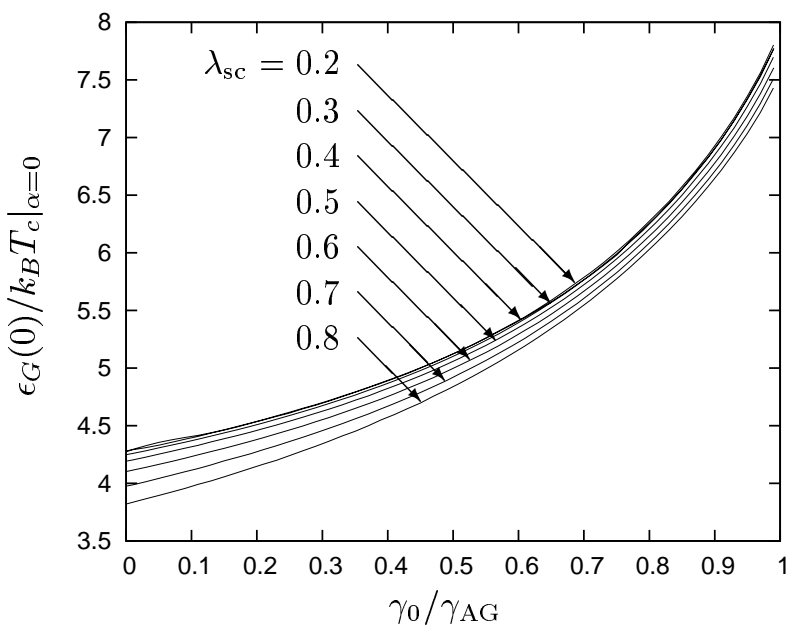

FIG. 3: $\epsilon_{G}(0) / k_{\mathrm{B}} T_{c}$ vs $\gamma_{0} / \gamma_{\mathrm{AG}}$ for $\lambda_{\mathrm{sc}}=0.2,0.3,0.4,0.5,0.6$, 0.7 , and 0.8 . In this figure, $\alpha=0$ is assumed.

When $\gamma_{0} / \gamma_{\mathrm{AG}} \simeq 1$, the ratio is as large as

$$
\frac{\epsilon_{G}(0)}{k_{\mathrm{B}} T_{c}} \simeq 8
$$

When $\alpha=0$, the ratio $\epsilon_{G}(0) / k_{\mathrm{B}} T_{c}$ is smaller than about 8 even in the limit of $\gamma_{0} \rightarrow \gamma_{\mathrm{AG}}$.

In general, the following relation holds:

$$
\frac{\epsilon_{G}(0)}{k_{\mathrm{B}} T_{c}}=\frac{\epsilon_{G}(0)}{k_{\mathrm{B}} T_{c}\left(\alpha=0, \gamma_{0}\right)} \frac{T_{c}\left(\alpha=0, \gamma_{0}\right)}{T_{c}\left(\alpha, \gamma_{0}\right)} .
$$

When $\alpha \neq 0$ and $\gamma_{0} / \gamma_{\mathrm{AG}} \lesssim 0.3$, it follows that

$$
\frac{\epsilon_{G}(0)}{k_{\mathrm{B}} T_{c}} \simeq\left(4+2 \frac{\gamma_{0}}{\gamma_{\mathrm{AG}}}\right)(1+\alpha)
$$

When $\alpha \neq 0$ and $\gamma_{0} / \gamma_{\mathrm{AG}} \simeq 1$, it follows that

$$
\frac{\epsilon_{G}(0)}{k_{\mathrm{B}} T_{c}} \simeq 8(1+2 \alpha)
$$

When $\alpha \gg 1$, the ratio $\epsilon_{G}(0) / k_{\mathrm{B}} T_{c}$ can be much larger than 8.

Figure 4 shows $\epsilon_{G}(T)$ as a function of $T$ for various $\alpha$ and $\gamma_{0}$ values. When $T=0 \mathrm{~K}, \epsilon_{G}(0)$ does not depend on $\alpha$ because $\alpha k_{\mathrm{B}} T=0$. When $\gamma_{0}$ is nonzero, not only $T_{c}$ but also $\epsilon_{G}(0)$ decreases; $\epsilon_{G}(0) / k_{\mathrm{B}} T_{c}$ remains nonzero and finite even in the limit of $\gamma_{0} \rightarrow \gamma_{\mathrm{AG}}$, as is shown in Fig. 3 and eq. (3.10). The amount of decrease in $\epsilon_{G}(T)$ with increasing $T$ is larger for a larger $\alpha$. In particular, $\left[d \epsilon_{G}(T) / d T\right]_{T=0 \mathrm{~K}}=0$ for $\alpha \lesssim 0.3$, but $\left[d \epsilon_{G}(T) / d T\right]_{T=0 \mathrm{~K}}<0$ apparently for $\alpha \gtrsim 0.3-0.7$.

Figure $5(\mathrm{a})$ shows the density of states $\rho(\varepsilon)$ or gap spectrum at $T=0 \mathrm{~K}$. A sharp coherence peak can only be seen for a sufficiently small $\gamma_{0}$ such as $\gamma_{0} / \gamma_{\mathrm{AG}} \ll 1$. The low-energy part of the density of states is expanded as

$$
\rho(\varepsilon)=\rho(0)+\rho^{(1)}\left|\frac{\varepsilon}{k_{\mathrm{B}} T_{c}}\right|+\frac{1}{2} \rho^{(2)}\left|\frac{\varepsilon}{k_{\mathrm{B}} T_{c}}\right|^{2}+\cdots,
$$

where $\rho^{(1)} / \rho_{0} \geq 0$. When $\gamma_{0} / \gamma_{\mathrm{AG}} \lesssim 0.3$, the gap spectrum has a convex-cup $\mathrm{V}$ shape, which is characterized by 


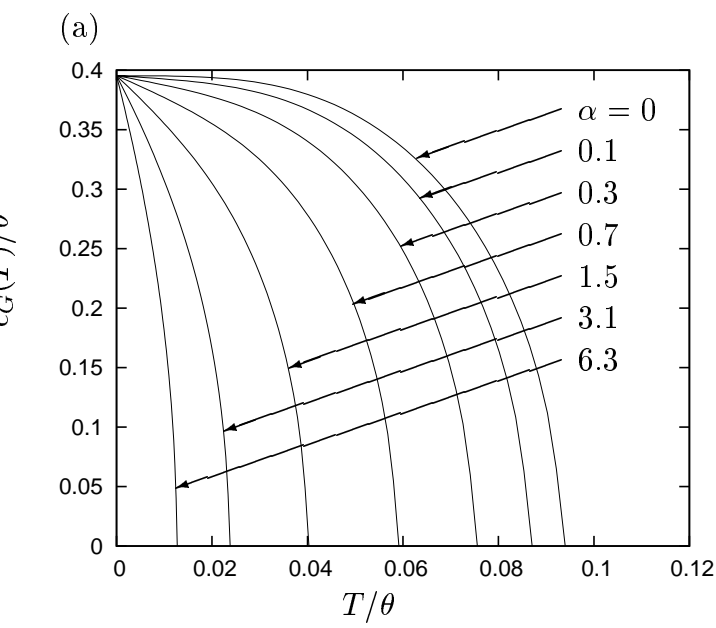

(c)

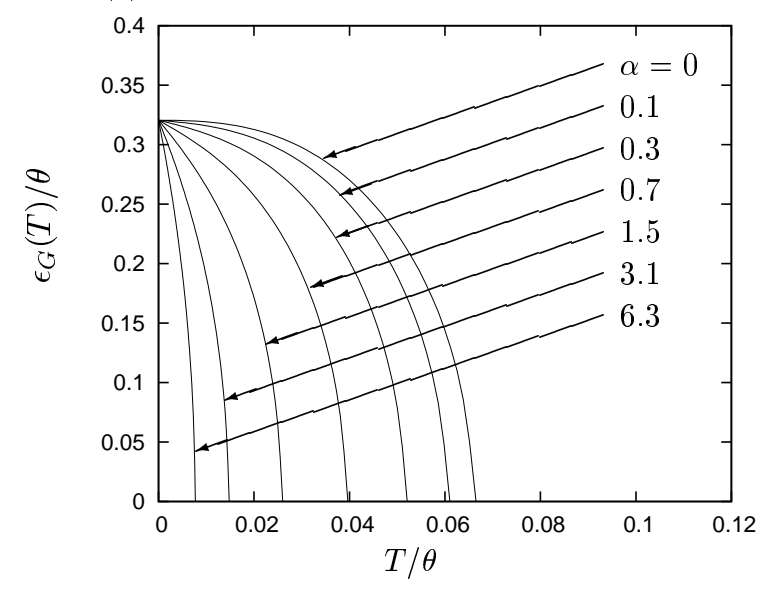

(b)

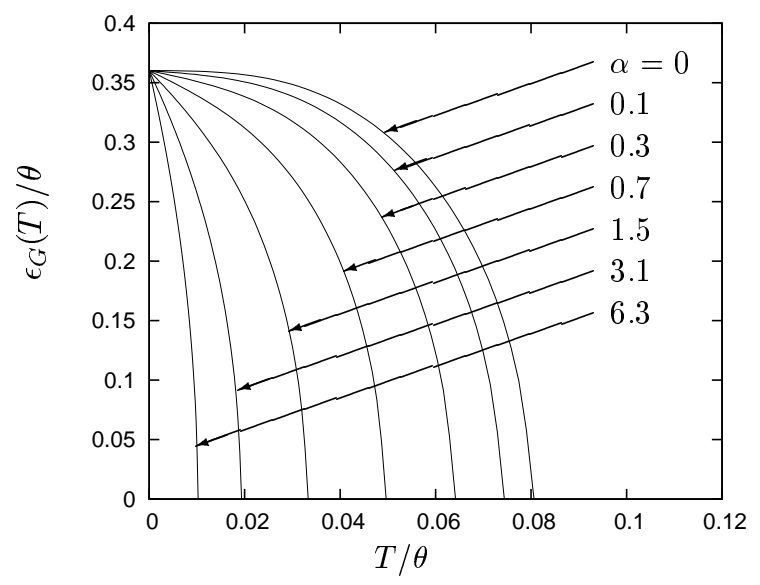

(d)

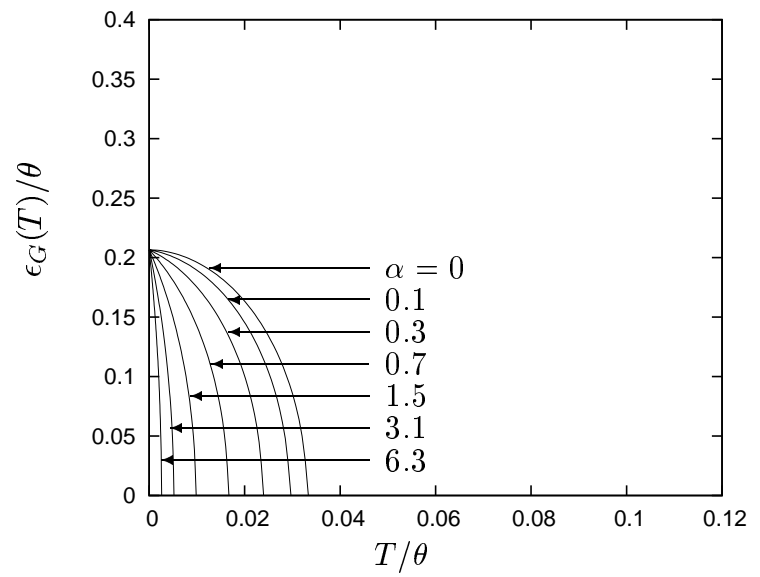

FIG. 4: $\epsilon_{G}(T)$ vs $T$ for (a) $\gamma_{0} / \gamma_{\mathrm{AG}}=0$, (b) 0.3 , (c) 0.6 , and (d) 0.9 . In each figure, results for $\alpha=0,0.1,0.3,0.7,1.5,3.1$, and 6.3 are shown.

(a)

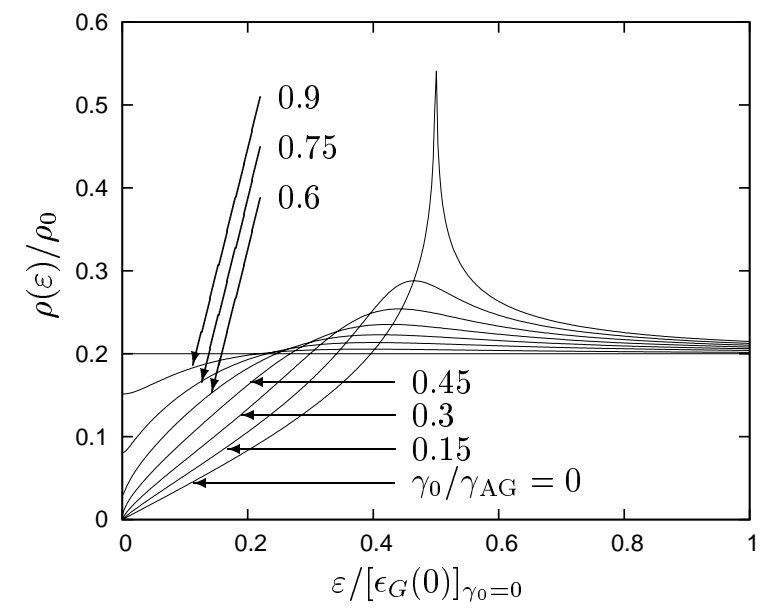

(b)

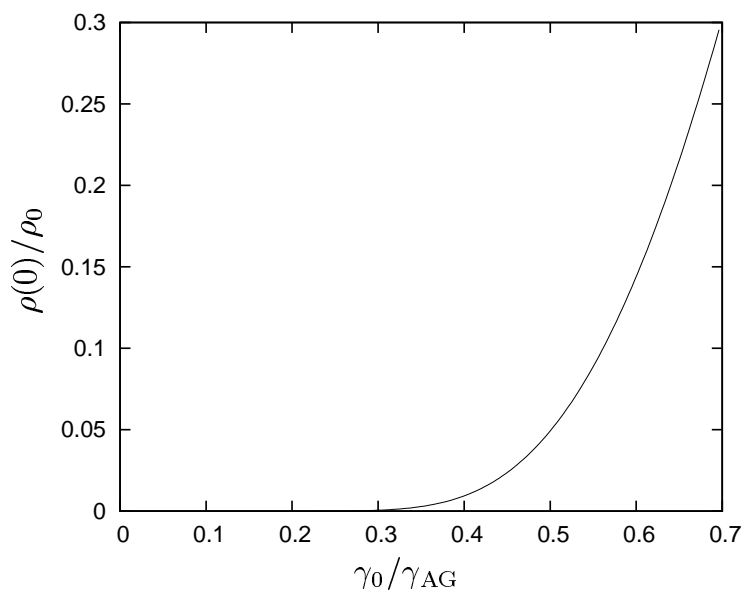

FIG. 5: (a) Density of states $\rho(\varepsilon)$ for $\gamma_{0} / \gamma_{\mathrm{AG}}=0,0.15,0.3,0.45,0.6,0.75$, and 0.9 and (b) $\rho(\varepsilon=0)$ at $T=0 \mathrm{~K}$ as a function of $\gamma_{0} / \gamma_{\mathrm{AG}}$. In the model of this paper, $\rho(\varepsilon)$ is an even function of $\varepsilon$.

$\rho^{(2)} / \rho_{0}>0$. In this case, $\rho(0)$ is negligible or at least almost negligible, as shown in Fig. $5(\mathrm{~b})$. When $\gamma_{0} / \gamma_{\mathrm{AG}} \simeq 0.3$, the gap spectrum has a straight-line $\mathrm{V}$ shape, which is characterized by $\rho^{(2)} / \rho_{0} \simeq 0 ; \rho(0)$ is also almost negligible. When $\gamma_{0} / \gamma_{\mathrm{AG}} \gtrsim 0.4-0.5$, the gap spectrum of $\rho(\varepsilon)$ has a concave- cap V shape, which is characterized by $\rho^{(2)} / \rho_{0}<0$. In this case, $\rho(0)$ is nonzero or the gap is a pseudo-gap. When $\gamma_{0} / \gamma_{\mathrm{AG}} \gtrsim 0.6$, in particular, $\rho(0)$ is significantly nonzero. A qualitatively similar result was obtained by Ueda and Rice for a $p$-wave superconductor with line zeros ${ }^{27,28}$. Ac- 
cording to ref. 27 , there is no critical $\gamma_{0} / \gamma_{\mathrm{AG}}$ for the onset of nonzero $\rho(0)$, i.e., $\rho(0)$ is nonzero when $\gamma_{0} / \gamma_{\mathrm{AG}}$ is nonzero. It is probable that, even in the model in this paper, $\rho(0)$ is nonzero when $\gamma_{0} / \gamma_{\mathrm{AG}}$ is nonzero, which is not inconsistent with Fig. 5(b). According to Fig. 5, however, there is practically a critical $\gamma_{0} / \gamma_{\mathrm{AG}}$ for the onset of a practically nonzero $\rho(0)$ and the qualitative change in the spectrum shape of $\rho(\varepsilon)$; when $\gamma_{0} / \gamma_{\mathrm{AG}} \lesssim 0.3, \rho(0)$ is nonzero but almost negligible and $\rho(\varepsilon)$ has a convex-cup $\mathrm{V}$ shape, while, when $\gamma_{0} / \gamma_{\mathrm{AG}} \gtrsim 0.3, \rho(0)$ is significantly nonzero and $\rho(\varepsilon)$ has a concave-cap $\mathrm{V}$ shape.

Figure 6 shows the density of states $\rho(\varepsilon)$ at $T>0 \mathrm{~K}$ for $\alpha=1$ and four values of $\gamma_{0} / \gamma_{\mathrm{AG}}$, namely, $0,0.3,0.5$, and 0.7 , for which a convex-cup $\mathrm{V}$ shape appears, a straightline $\mathrm{V}$ shape appears, a concave-cap $\mathrm{V}$ shape appears, and not only a concave-cap $\mathrm{V}$ shape appears but also $\rho(0)$ is large, respectively, at $T=0 \mathrm{~K}$. When $T>0 \mathrm{~K}, \rho(0)$ is nonzero and $\rho(\varepsilon)$ has a convex-cup shape near the chemical potential.

\section{Application to Cuprate Superconductors}

The specific heat coefficient is given by

$$
\gamma_{\mathrm{C}}=\frac{2}{3} \pi^{2} k_{\mathrm{B}}^{2} \rho^{*}(0),
$$

where $\rho^{*}(0)$ is the density of states of quasi-particles at the chemical potential. The observed $\gamma_{\mathrm{C}}$ is as large as

$$
\gamma_{\mathrm{C}} \simeq(10-15) \mathrm{mJ} / \mathrm{mol} \cdot \mathrm{K}^{2},
$$

for $\delta \simeq \delta_{\text {opt }} \cdot{ }^{29,30}$ Then, it follows that

$$
\rho^{*}(0) \simeq 3 \times 10^{-3} \text { states } / \mathrm{meV},
$$

in optimally doped cuprate oxides; $\gamma_{\mathrm{C}}$ and $\rho^{*}(0)$ are smaller than those in eqs. (4.2) and (4.3), respectively, in underdoped cuprate oxides.

In the two-dimensional free-electron model, the twodimensional resistivity $R_{\square}$ is given by

$$
R_{\square}=\frac{m^{*}}{n_{\square} e^{2} \tau_{\mathrm{tr}}},
$$

where $m^{*}$ is the effective mass, $n_{\square}$ is the two-dimensional electron density, and $\tau_{\mathrm{tr}}$ is the relaxation time relevant to conductivity. The density of states for quasi-particles is given by

$$
\rho^{*}(\varepsilon)=\frac{a^{2} m^{*}}{2 \pi \hbar}
$$

It follows from eqs. (4.4) and (4.5) that

$$
R_{\square}=\frac{4 \pi \hbar}{e^{2}} \frac{1}{n} \rho^{*}(0) \frac{\hbar}{2 \tau_{\mathrm{tr}}},
$$

where $n=n_{\square} a^{2}$ is the two-dimensional electron density per unit cell. Three-dimensional resistivity is given by

$$
R=\frac{4 \pi \hbar c}{e^{2}} \frac{1}{n} \rho^{*}(0) \frac{\hbar}{2 \tau_{\mathrm{tr}}},
$$

where $c$ is the lattice constant perpendicular to $\mathrm{CuO}_{2}$ planes or the average distance between nearest-neighbor $\mathrm{CuO}_{2}$ planes. When $c=4 \AA$ is assumed,

$$
\frac{4 \pi \hbar}{e^{2}}=51.6 \mathrm{k} \Omega
$$

so that

$$
\frac{4 \pi \hbar c}{e^{2}}=2.1 \times 10^{3} \mu \Omega \mathrm{cm} .
$$

If scatterings are homogeneous and isotropic, the relaxation time appearing in the conductivity is simply given by the relaxation time or lifetime of quasi-particles such that

$$
\begin{aligned}
\frac{\hbar}{2 \tau_{\mathrm{tr}}} & =-\operatorname{Im} \Sigma(+\mathrm{i} 0 ; T) \\
& =\gamma_{0}+\alpha k_{\mathrm{B}} T,
\end{aligned}
$$

above $T_{c}$. When forward scatterings are strong, $\hbar / 2 \tau_{\operatorname{tr}}<$ $-\operatorname{Im} \Sigma(+\mathrm{i} 0 ; T)$ in general. When eq. (4.10) is assumed,

$$
R(T) \simeq \frac{4 \pi \hbar c}{e^{2}} \rho^{*}(0)\left(\gamma_{0}+\alpha k_{\mathrm{B}} T\right),
$$

where $n \simeq 1$ is assumed.

In optimally doped cuprates, the ratio $\epsilon_{G}(0) / k_{\mathrm{B}} T_{c}$ is as large as 8 . It is substantially larger than its mean-field value of about $4,{ }^{21,22}$ or those shown in Fig. 3 of this paper. Since the residual resistivity is rather small in optimally doped cuprates, elastic scatterings are supposed to be weak or $\gamma_{0} \simeq 0$, which is consistent with the observed high $T_{c}$. When inelastic scatterings are effective or $\alpha \neq 0$, it follows according to eq. (3.10) that

$$
\frac{\epsilon_{G}(0)}{k_{\mathrm{B}} T_{c}} \simeq 4(1+\alpha) .
$$

The observed large ratio can be explained if $\alpha \simeq 1$. According to eqs. (1.2), (4.3), and (4.11), on the other hand, it follows that $\alpha \simeq 1$. The large $T$-linear coefficient of resistivity, which is as large as $r_{1} \simeq 1 \mu \Omega \mathrm{cm} / \mathrm{K}$, as is given by eq. (1.2), and the large ratio $\epsilon_{G}(0) / k_{\mathrm{B}} T_{c} \simeq 8$ are consistent with each other.

The resistivity extrapolated to $T \rightarrow 0 \mathrm{~K}$ or residual resistivity is given by

$$
R(0) \simeq \frac{4 \pi \hbar c}{e^{2}} \rho^{*}(0) \gamma_{0},
$$

for $n \simeq 1$. Experimentally, $T_{c}$ decreases almost linearly in the residual resistivity $R(0)$ in underdoped cuprates, ${ }^{31,32}$ which is inconsistent with the AG theory or the curve for $\alpha=0$ in Fig. 1 in this paper. According to the analysis given in this paper, when $\alpha \gtrsim 1, T_{c}$ decreases almost linearly with the residual resistivity. This is also consistent with the fact that inelastic scatterings by superconducting and antiferromagnetic fluctuations are crucial in underdoped cuprate superconductors.

When $\delta \simeq 0.05$, where $T_{c}$ is negligible, the residual resistivity is as large as $R(0) \simeq 10^{3} \mu \Omega \mathrm{cm}$. This leads to the argument that the critical pair breaking for $\delta \simeq 0.05$ must be as large as $\gamma_{\mathrm{AG}} \simeq 10^{2} \mathrm{meV}$ so that $T_{c 0} \simeq 10^{3} \mathrm{~K}$ for $\delta \simeq 0.05$. However, this seems to be unreasonable since $T_{c 0}$ 
(a)

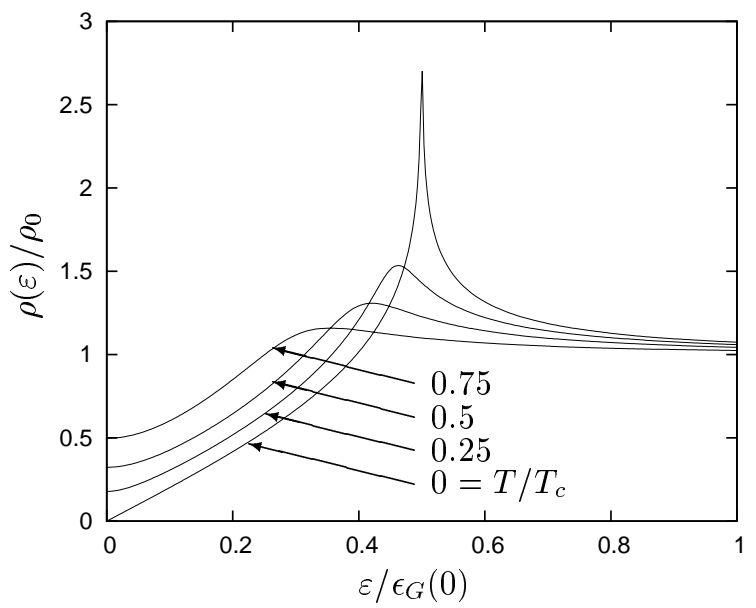

(c)

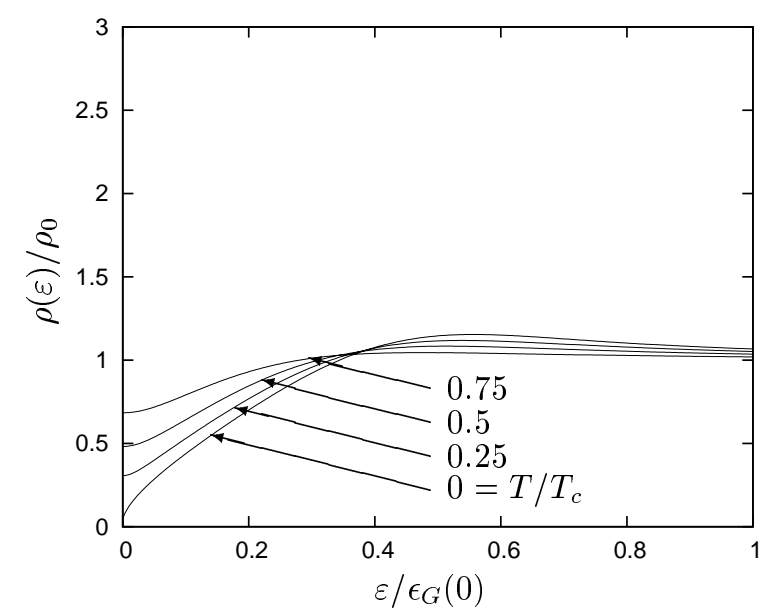

(b)

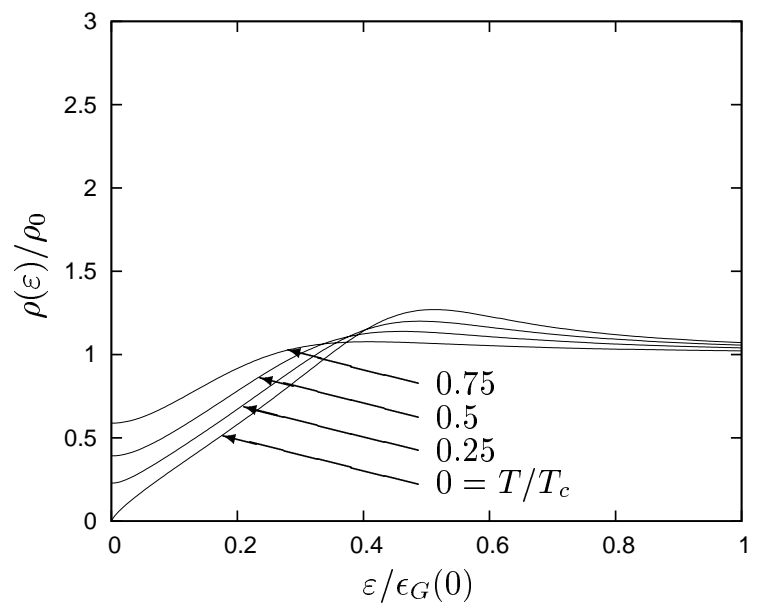

(d)

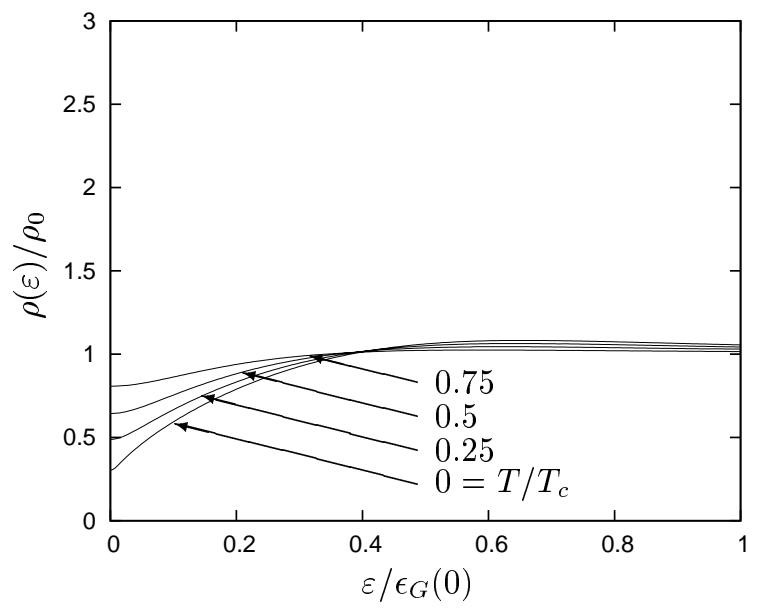

FIG. 6: Temperature dependence of density of states for $\alpha=1$ : (a) $\gamma_{0} / \gamma_{\mathrm{AG}}=0$, (b) 0.3 , (c) 0.5, and (d) 0.7 . In all the figures, results for $T / T_{c}=0,0.25,0.5$ and 0.75 are shown.

seems to be too high. One of the possible explanations is that the crucial assumption on the impurity strength and distribution, which is discussed in $\S 2$, is not valid for underdoped cuprate oxide superconductors. Inhomogeneous disorder is presumably responsible for this discrepancy such that superconductivity is possible in a region where $\gamma_{0}$ is small and that it disappears in other regions where $\gamma_{0}$ is large. It will be interesting to theoretically examine how large the final resistivity of such an inhomogeneously disordered system is.

When $\gamma_{0} / \gamma_{\mathrm{AG}} \gtrsim 0.4-0.5, \rho(0)$ is nonzero and the lowenergy part is of convex-cup $\mathrm{V}$ shape, as shown in Fig. 5(a). The nonzero $\rho(0)$ means that the specific heat has a $T$ linear term in the limit of $T \rightarrow 0 \mathrm{~K}$ such that

$$
C=\gamma_{\mathrm{C}} T+O\left(T^{2}\right)
$$

where $\gamma_{\mathrm{C}}$ is nonzero. On the other hand, the $T$-linear specific heat coefficient $\gamma_{C}$ is also nonzero in an inhomogeneous superconducting state, in which some regions are superconducting and other regions are normal. Since the pair breaking may be weak in superconducting regions, the gap structure in the inhomogeneous superconducting state is not necessarily of concave-cap $\mathrm{V}$ shape even if the observed $\gamma_{C}$ is nonzero. Whether the gap spectrum is of concave-cap or convex-cup $\mathrm{V}$ shape can be used as a criterion for determining the origin of the nonzero $\gamma_{C}$ : inhomogeneity or nonzero $\rho(0)$. It will be interesting to search for a gap spectrum of concave-cap $\mathrm{V}$ shape in an anisotropic superconductor with a nonzero $T$-linear specific heat coefficient.

In addition to disorders that inevitably exist in cuprate oxide, nonmagnetic and magnetic impurities such as Zn and $\mathrm{Ni}$ are often introduced into cuprate oxide. The scattering by a single impurity of $\mathrm{Ni}$ or $\mathrm{Zn}$ is as strong as the unitaritylimit scattering. The treatment discussed in this paper cannot apply in such a case. Hotta ${ }^{33}$ and Sun and Maki ${ }^{34}$ considered such a case in $T$-matrix approximation under an assumption that resonance occurs in the scattering process at the chemical potential. In their treatments, no concavecap $\mathrm{V}$ shape appears because the resonance is crucial to obtain a nonzero and large $\rho(0)$. It will be interesting to study how a crossover occurs between a convex-cup V shape under the resonance condition, i.e., when a single impurity is as strong as a unitarity-limit scatterer and the impurity concentration is low, and a concave-cap V shape under an off-resonance condition, i.e., when a single impurity is weak and the impurity concentration is high. 


\section{Conclusion}

We study the effects of elastic and inelastic scatterings on $d$-wave superconductivity in optimally doped and underdoped cuprate oxides. Since the observed resistivity increases almost linearly as a function of temperature, it is reasonable to assume the existence of a $T$-linear term in the lifetime width of quasi-particles such as $\hbar / 2 \tau \simeq k_{\mathrm{B}} T$. Then, the Abrikosov-Gor'kov theory is modified in such a way that the superconducting critical temperature $T_{c}$ decreases almost linearly as a function of impurity concentration. The deviation from the Abrikosov-Gor'kov theory is not evidence of the fact that high- $T_{c}$ superconductivity is realized by an exotic mechanism. If elastic scatterings by impurities are ignored, the ratio $\epsilon_{G}(0) / k_{\mathrm{B}} T_{c}$ is about 8 , which is twice as large as its value of about 4 predicted by the mean-field theory. If elastic scatterings are considered, the ratio can be much larger than 8 . When elastic scatterings by impurities are sufficiently strong, the density of states $\rho(\varepsilon)$ not only is significantly nonzero at the chemical potential such as $\rho(0)>0$ but also has a concave-cap $\mathrm{V}$ shape in the low-energy part at least at the zero temperature. If the $T$-linear specific heat coefficient is significantly nonzero in an underdoped cuprate-oxide superconductor, it will be interesting to search for the density of states $\rho(\varepsilon)$ of the superconductor for such a concave-cap V shape.

\section{Acknowledgements}

The authors are grateful to M. Ido, M. Oda, and N. Momono for helpful discussions on various properties of cuprate oxide superconductors.
1 J. G. Bednortz and K. A. Müller: Z. Phys. B 64 (1986) 189.

2 D. J. Van Harlingen: Rev. Mod. Phys. 67 (1995) 515.

3 J. Orenstein and A. J. Mills: Science 288 (2000) 468.

${ }^{4}$ S. A. Kivelson, I. P. Bindloss, E. Fradkin, V. Oganesyan, J. M. Tranquada, A. Kapitulnik, and C. Howald: Rev. Mod. Phys. 75 (2003) 1201.

${ }^{5}$ P. A. Lee, N. Nagaosa, and X-G. Wen: Rev. Mod. Phys. 78 (2006) 17.

6 O. Fischer, M. Kugler, I. Maggio-Aprile, C. Berthod, and C. Renner: Rev. Mod. Phys. 79 (2007) 353.

7 K. B. Lyons, P. A. Fleury, L. F. Schneemeyer, and J. V. Waszczak: Phys. Rev. Lett. 60 (1988) 732.

${ }^{8}$ H. Takagi, B. Batlogg, H. L. Kao, J. Kwo, R. J. Cava, J. J. Krajewski, and W. F. Peck, Jr.: Phys. Rev. Lett. 69 (1992) 2975.

9 B. Batlogg, H. Y. Hwang, H. Takagi, H. L. Kao, J. Kwo, and R. J. Cava: J. Low Temp. Phys. 95 (1994) 23.

10 T. Moriya, Y. Takahashi, and K. Ueda: J. Phys. Soc. Jpn. 59 (1990) 2905.

11 A. A. Abrikosov and L. P. Gor'kov: Sov. Phys. JETP 12 (1961) 1243.

12 J. Bardeen, L. Cooper, and J. R. Schrieffer: Phys. Rev. 108 (1957) 1175

13 T. Tsuneto: Prog. Theor. Phys. 28 (1962) 857.

14 V. L. Pokrovskii and M. S. Ryvkin: Sov. Phys. JETP 16 (1963) 67.

15 D. Markowitz and L. P. Kadanoff: Phys. Rev. 131 (1963) 563.

16 S. Nakajima: Prog. Theor. Phys. 32 (1964) 871.

17 M. D. Whitmore and J. P. Carbotte: Phys. Rev. B 23 (1981) 5782.

18 F. J. Ohkawa and H. Fukuyama: J. Phys. Soc. Jpn. 53 (1984)
4344 .

19 S. Skalski, O. Betbeder-Matibet, and P. R. Weiss: Phys. Rev. 136 (1964) A1500.

${ }^{20}$ L. S. Borkowski and P. J. Hirschfeld: Phys. Rev. B 49 (1994) 15404.

${ }^{21}$ F. J. Ohkawa: J. Phys. Soc. Jpn. 56 (1987) 2267.

${ }^{22}$ K. Maki and H. Won: Phys. Rev. Lett. 72 (1994) 1758.

${ }^{23}$ F. J. Ohkawa and T. Toyama: cond-mat, arXiv:0901.1410.

${ }^{24}$ F. J. Ohkawa: J. Phys. Soc. Jpn. 78 (2009) 084712.

25 At these averages, $V_{i}=0$ and $J_{i}=0$ are assumed for nonimpurity sites, whereas $V_{i} \neq 0$ or $J_{i} \neq 0$ is assumed for impurity sites.

${ }^{26}$ When a superconducting gap opens, antiferromagnetic and superconducting fluctuations are suppressed so that the lifetime width due to inelastic scatterings is reduced below $T_{c}$. The assumption simply means that the reduction in the lifetime width below $T_{c}$ is negligible.

${ }^{27}$ K. Ueda and T. M. Rice: Theory of Heavy Fermions and Valence Fluctuations ed. T Kasuya and T. Saso, Springer Series in Solid-State Sciences (Springer-Verlag, Berlin, 1985) 62, p. 267.

28 M. Sigrist and K. Ueda: Rev. Mod. Phys. 63 (1991) 239.

29 J. W. Loram, K. A. Mirza, J. R. Cooper, and W. Y. Liang: Phys. Rev. Lett. 71 (1993) 1740.

30 N. Momono and M. Ido: Physica C 264 (1996) 311.

31 Y. Fukuzumi, K. Mizuhashi, K. Takenaka, and S. Uchida: Phys. Rev. Lett. 76 (1996) 684.

${ }^{32}$ F. Rullier-Albenque, H. Alloul, and R. Tourbot: Phys. Rev. Lett 91 (2003) 047001.

33 T. Hotta: J. Phys. Soc. Jpn. 62 (1993) 274

${ }^{34}$ Y. Sun and K. Maki: Phys. Rev. B 51 (1995) 6059 